\title{
N-TUPLES OF POSITIVE INTEGERS WITH THE SAME SUM AND THE SAME PRODUCT
}

\author{
YONG ZHANG AND TIANXIN CAI
}

\begin{abstract}
In this paper, by using the theory of elliptic curves, we prove that for every $k$, there exists infinitely many primitive sets of $k n$-tuples of positive integers with the same sum and the same product.
\end{abstract}

\section{INTRODUCTION}

In 1981, J. G. Mauldon 3 proposed the Problem E2872: Find five different triples of positive integers with the same sum and the same product. In 1982, L. L. Foster and G. Robins [1] gave ten triples with the sum 132600 and the product $2^{7} 3^{6} 5^{4} 7^{2} 13^{3} 17^{3}$. In 1996 , A. Schinzel [4] proved that for every $k$, there exist infinitely many primitive sets of $k$ triples of positive integers with the same sum and the same product, i.e., the system of equations

$$
\left\{\begin{array}{l}
x_{i 1}+x_{i 2}+x_{i 3}=A, \\
x_{i 1} x_{i 2} x_{i 3}=B, \\
x_{i j}>0, A>0, B>0 \\
i=1, \cdots, k, j=1,2,3
\end{array}\right.
$$

has $k$ solutions for every $k \in \mathbb{N}-\{0\}$. A set $S$ of triples is called primitive if the greatest common divisor of all elements of all triples of $S$ is 1 .

More information on this problem can be found in [2]: D16 Triples with the same sum and the same product and D24 Sum equals product.

In this paper, we consider the generalized system of equations

$$
\left\{\begin{array}{l}
x_{i 1}+\cdots+x_{i n}=A \\
x_{i 1} \cdots x_{i n}=B \\
x_{i j}>0, A>0, B>0, \\
i=1, \cdots, k, j=1, \cdots, n, n \geq 4 .
\end{array}\right.
$$

By using the theory of elliptic curves, we prove the following theorem.

Theorem 1.1. For every $k$, there exist infinitely many primitive sets of $k n$-tuples of positive integers with the same sum and the same product.

Received by the editor April 20, 2011 and, in revised form, August 19, 2011.

2010 Mathematics Subject Classification. Primary 11D25; Secondary 11D72, 11 G05.

Key words and phrases. $n$-tuples, the system of equations, elliptic curves.

This research was supported by China National Science Foundation Grant No.10871169. 


\section{An important LEMMA}

In [4, A. Schinzel proved that:

The system of equations

$$
x_{1}+x_{2}+x_{3}=x_{1} x_{2} x_{3}=6
$$

has infinitely many solutions in rational numbers $x_{j}>0$.

The equation

$$
x_{1}+\cdots+x_{n}=x_{1} \cdots x_{n}, n \geq 3
$$

always has at least one solution $(1,1, \cdots, 1,2, n)$ such that it has the same sum and product $2 n$.

To prove the theorem, we consider an analogous system of equations and get the following lemma.

Lemma 2.1. The system of equations

$$
x_{1}+\cdots+x_{n}=x_{1} \cdots x_{n}=2 n
$$

has infinitely many solutions in rational numbers $x_{j}>0$ for $n \geq 3$.

Proof. Because of Schinzel's result, we can assume that $n \geq 4$. Taking $x_{1}=$ $1, \cdots, x_{n-3}=1$, we have

$$
\left\{\begin{array}{l}
x_{n-2}+x_{n-1}+x_{n}=n+3, \\
x_{n-2} x_{n-1} x_{n}=2 n .
\end{array}\right.
$$

Eliminating $x_{n}$ of $(2.2)$, we get

$$
x_{n-2}^{2} x_{n-1}+x_{n-2} x_{n-1}^{2}-(n+3) x_{n-2} x_{n-1}+2 n=0,
$$

leading to

$$
\left(\frac{x_{n-2}}{x_{n-1}}\right)^{2}+\frac{x_{n-2}}{x_{n-1}}-(n+3) \frac{x_{n-2}}{x_{n-1}} \frac{1}{x_{n-1}}+2 n\left(\frac{1}{x_{n-1}}\right)^{3}=0 .
$$

Taking

we have

$$
u=\frac{x_{n-2}}{x_{n-1}}, v=\frac{1}{x_{n-1}},
$$

$$
u^{2}+u-(n+3) u v+2 n v^{3}=0
$$

Let

$$
y=216 n(2 u+1-(n+3) v), x=-72 n v+3(n+3)^{2},
$$

and we get

$$
\begin{aligned}
E_{n}: y^{2}= & x^{3}-27(n+3)\left(n^{3}+9 n^{2}-21 n+27\right) x+54 n^{6}+972 n^{5}+3402 n^{4} \\
& -5832 n^{3}+7290 n^{2}-26244 n+39366 .
\end{aligned}
$$

This is a family of elliptic curves, which is defined over $\mathbb{Q}$. We study the rational points on $E_{n}$.

The discriminant of $E_{n}$ is $\Delta=2^{15} 3^{12} n^{3}\left(n^{3}+9 n^{2}-27 n+27\right)$, and when $n \geq 4$, we have $\Delta>0$, this means that $E_{n}$ is nonsingular. Meanwhile, the cubic equation

$$
\begin{aligned}
& x^{3}-27(n+3)\left(n^{3}+9 n^{2}-21 n+27\right) x \\
& +54 n^{6}+972 n^{5}+3402 n^{4}-5832 n^{3}+7290 n^{2}-26244 n+39366=0
\end{aligned}
$$


has three different real roots $x_{1}(n), x_{2}(n), x_{3}(n)$. Suppose $x_{1}(n)<x_{2}(n)<x_{3}(n)$, then from the relationship of roots and coefficients, we have

$$
\left\{\begin{array}{l}
x_{1}(n)+x_{2}(n)+x_{3}(n)=0, \\
x_{1}(n) x_{2}(n) x_{3}(n)=-f(n),
\end{array}\right.
$$

where $f(n)=54 n^{6}+972 n^{5}+3402 n^{4}-5832 n^{3}+7290 n^{2}-26244 n+39366$. An easy calculation shows that $f(n)>0$ when $n \geq 4$, then

$$
x_{1}(n)<0<x_{2}(n)<x_{3}(n),-x_{1}(n)=x_{2}(n)+x_{3}(n) .
$$

It is easy to check that the points $P=\left(3(n+3)^{2}, 216 n\right), Q=\left(3(n-3)^{2}, 108 n(n-\right.$ $1))$ and $R=\left(3(n+3)^{2}-72 n, 216 n(n-2)\right)$ lie on $E_{n}$. Using the Group Law on elliptic curves, we obtain the points

$$
[2] P=O, P+Q+R=O
$$

and

$$
\begin{aligned}
{[2] R=} & \left(\frac{3\left(n^{4}+2 n^{3}+13 n^{2}-36 n+36\right)}{(n-2)^{2}},-\frac{216\left(2 n^{3}-6 n^{2}+7 n-2\right)}{(n-2)^{3}}\right), \\
{[3] R=} & \left(3\left(n^{6}-36 n^{4}+126 n^{3}-180 n^{2}+108 n-15\right) /\left(n^{2}-3 n+3\right)^{2},\right. \\
& \left.108(n-1)(n-2)\left(7 n^{4}-33 n^{3}+67 n^{2}-66 n+28\right) /\left(n^{2}-3 n+3\right)^{3}\right),
\end{aligned}
$$

where $O$ denotes the point at infinity on $E_{n}$ and $[m]$ is the isogeny multiplication by $m$, which is defined by $[m](P)=P+\cdots+P$ ( $m$ terms $)$. This means that $P$ is a point of order 2 and $P, Q, R$ lie on a line.

To prove that there are infinitely many rational points on $E_{n}$, it is enough to find a point on $E_{n}$ with $x$-coordinate not in $\mathbb{Z}$. When the numerator of the $x$-coordinate of [3] $R$ is divided by $\left(n^{2}-3 n+3\right)^{2}$, the remainder equals $r=-36\left(3 n^{3}-12 n^{2}+\right.$ $18 n-10)$ and $r \neq 0$ when $n \geq 4$, so the $x$-coordinate of [3] $R$ is not a polynomial. For $4 \leq n \leq 109$ one can check that $r /\left(n^{2}-3 n+3\right)^{2}$ is not an integer, and that it is nonzero and less than 1 in modulus for $n>109$. Hence for all $n \geq 4$ the point [3] $R$ has nonintegral $x$-coordinate and hence, by the Nagell-Lutz Theorem (see p. 56 of [5]), is of infinite order. Then there are infinitely many rational points on $E_{n}$.

From the transformation (2.3), we have

$$
u=\frac{y-3 x n-9 x+9 n^{3}+81 n^{2}+27 n+243}{432 n}, v=\frac{3(n+3)^{2}-x}{72 n},
$$

leading to

$$
x_{n-2}=\frac{y-3 x n-9 x+9 n^{3}+81 n^{2}+27 n+243}{6\left(-x+3 n^{2}+18 n+27\right)}, x_{n-1}=\frac{72 n}{3(n+3)^{2}-x} ;
$$

then

$$
x_{n}=n+3-x_{n-2}-x_{n-1}=\frac{-y-3 x n-9 x+9 n^{3}+81 n^{2}+27 n+243}{6\left(-x+3 n^{2}+18 n+27\right)} .
$$


Therefore,

$$
\begin{aligned}
& \left(x_{1}, \cdots, x_{n-3}, x_{n-2}, x_{n-1}, x_{n}\right) \\
& =\left(1, \cdots, 1, \frac{y-3 x n-9 x+9 n^{3}+81 n^{2}+27 n+243}{6\left(-x+3 n^{2}+18 n+27\right)},\right. \\
& \left.\frac{72 n}{3(n+3)^{2}-x}, \frac{-y-3 x n-9 x+9 n^{3}+81 n^{2}+27 n+243}{6\left(-x+3 n^{2}+18 n+27\right)}\right)
\end{aligned}
$$

is a solution of $(2.1)$.

In view of $x_{j}>0, j=1, \cdots, n$, we have the condition

$$
x<3(n+3)^{2},|y|<-3 x n-9 x+9 n^{3}+81 n^{2}+27 n+243 .
$$

From the graph of $|y|=-3 x n-9 x+9 n^{3}+81 n^{2}+27 n+243$, it is easy to see that when

$$
x<\frac{3\left(n^{3}+9 n^{2}+3 n+27\right)}{n+3},
$$

the above condition is satisfied, because

$$
\frac{3\left(n^{3}+9 n^{2}+3 n+27\right)}{(n+3)}-3(n+3)^{2}=-\frac{72 n}{(n+3)}<0
$$

for $n \geq 4$, and

$$
|y|=g\left(\frac{3\left(n^{3}+9 n^{2}+3 n+27\right)}{n+3}\right)=0,
$$

where $g(x)=-3 x n-9 x+9 n^{3}+81 n^{2}+27 n+243$.

Since $|y|=-3 x n-9 x+9 n^{3}+81 n^{2}+27 n+243$ is the equation of the pair of tangent at $P=\left(3(n+3)^{2}, 216 n\right)$ and $-P=\left(3(n+3)^{2},-216 n\right)$, they intersect at the point

$$
\left(\frac{3\left(n^{3}+9 n^{2}+3 n+27\right)}{n+3}, 0\right) .
$$

It is easy to see that

$$
x_{2}(n)<\frac{3\left(n^{3}+9 n^{2}+3 n+27\right)}{n+3}<x_{3}(n) .
$$

In virtue of the theorem of Poincaré and Hurwitz (see [6], Chap. V, p. 78, Satz 11), $E_{n}$ has infinitely many rational points in every neighborhood of any one of them. The point [3] $R$ satisfies the inequality $|y|<-3 x n-9 x+9 n^{3}+81 n^{2}+27 n+243$, since

$$
\begin{aligned}
& \frac{3\left(n^{6}-36 n^{4}+126 n^{3}-180 n^{2}+108 n-15\right)}{\left(n^{2}-3 n+3\right)^{2}}-\frac{3\left(n^{3}+9 n^{2}+3 n+27\right)}{n+3} \\
& =\frac{-36\left(3 n^{2}-7 n+6\right)\left(3 n^{2}-6 n+4\right)}{\left(n^{2}-3 n+3\right)^{2}(n+3)}<0,
\end{aligned}
$$

when $n \geq 4$. Hence, there are infinitely many rational points of $E_{n}$ satisfying $|y|<-3 x n-9 x+9 n^{3}+81 n^{2}+27 n+243$. Therefore, we can find infinitely many solutions in rational numbers $x_{j}>0, j=1, \cdots, n$ satisfying (2.1).

As an example, when $n=4$, we have $R=(-141,1728),[2] R=(363,-6264)$, [3] $R=(1443 / 49,334368 / 343)$. 


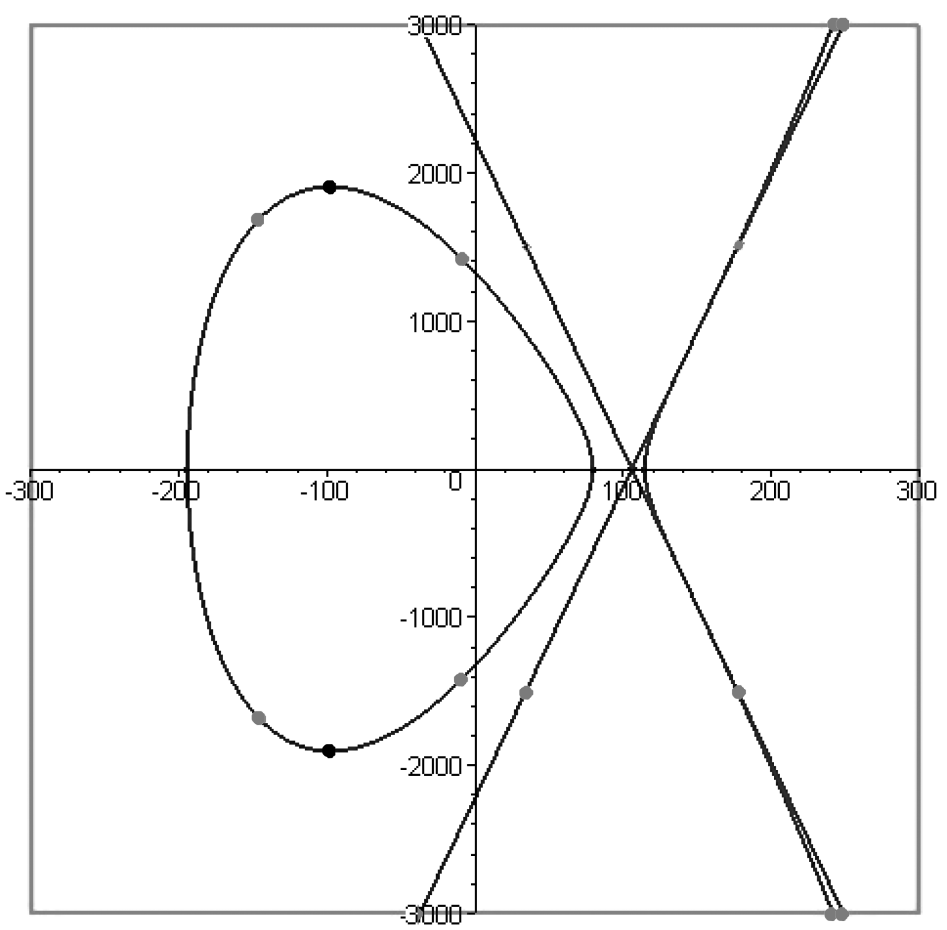

FiguRE 1. $E_{4}: y^{2}=x^{3}-28539 x+1765206$ and $|y|=2223-21 x$

From the transformation (2.3), we have

$$
u=\frac{y+6048 x-864}{1728}, v=\frac{147-x}{288},
$$

leading to

$$
x_{2}=\frac{21 x-2223-y}{6(x-147)}, x_{3}=\frac{288}{147-x}
$$

then

$$
x_{4}=8-1-x_{2}-x_{3}=\frac{21 x-2223+y}{6(x-147)} .
$$

Therefore,

$$
\left(x_{1}, x_{2}, x_{3}, x_{4}\right)=\left(1, \frac{21 x-2223-y}{6(x-147)}, \frac{288}{147-x}, \frac{21 x-2223+y}{6(x-147)}\right)
$$

is a solution of (2.1) for $n=4$.

In Figure1, we display the elliptic curve $E_{4}$ and the lines $|y|=2223-21 x$. It is easy to see that if $(x, y)$ on $E_{4}$ and $x \leq x_{2}(4)$, we have $|y|<2223-21 x$, then there are infinitely many solutions in rational numbers $x_{j}>0, j=1, \cdots, 4$ for (2.1). By an easy calculation, we have the following examples. The points

$$
(x, y)=(3,1296),\left(\frac{1443}{49}, \frac{334368}{343}\right),\left(\frac{-3423813}{34969}, \frac{12443156928}{6539203}\right)
$$


lead to

$$
\left(x_{1}, x_{2}, x_{3}, x_{4}\right)=(1,4,2,1),\left(1, \frac{49}{20}, \frac{128}{35}, \frac{25}{28}\right),\left(1, \frac{103058}{24497}, \frac{34969}{29737}, \frac{68644}{42449}\right),
$$

respectively.

\section{Proof of the theorem}

Proof of Theorem 1.1. The method is as used in [4. Take any $k$ positive rational solutions $\left(x_{i 1}, \cdots, x_{i n}\right)$, where $x_{i 1}=1, \cdots, x_{i, n-3}=1$, of $(2.1)$. Let $d=l c m_{i, j}\left(x_{i j}, j=\right.$ $1, \cdots, n, i \leq k)$, we set

$$
x_{i j}=\frac{a_{i j}}{d}, a_{i j} \in \mathbb{N}-\{0\},\left(\operatorname{gcd}_{i, j}\left(a_{i j}\right), d\right)=1,
$$

where $a_{i 1}=d, \cdots, a_{i, n-3}=d$. Then

$$
\sum_{i=1}^{n} a_{i j}=2 n d, \prod_{i=1}^{n} a_{i j}=2 n d^{n}(i \leq k),
$$

hence

$$
\operatorname{gcd}_{i, j}\left(a_{i j}\right)=1 .
$$

For two sets of solutions $\left\{\left(x_{i 1}, \cdots, x_{i n}\right), i \leq k\right\}$ and $\left\{\left(x_{i 1}^{\prime}, \cdots, x_{i n}^{\prime}\right), i \leq k\right\}$, if the sets of $n$-tuples $\left\{\left(a_{i 1}, \cdots, a_{i n}\right), i \leq k\right\}$ and $\left\{\left(a_{i 1}^{\prime}, \cdots, a_{i n}^{\prime}\right), i \leq k\right\}$ coincides, then we have $d=d^{\prime}$ by (3.1). Hence, the sets of solutions themselves coincide. Since there are infinitely many choices of $k$ elements from an infinite set, then for every $k$ there exist infinitely many primitive sets of $k n$-tuples of positive integers with the same sum and the same product.

Example 1. For $n=4$, we have three rational quadruples

$$
(1,4,2,1),\left(1, \frac{49}{20}, \frac{128}{35}, \frac{25}{28}\right),\left(1, \frac{103058}{24497}, \frac{34969}{29737}, \frac{68644}{42449}\right) .
$$

Then $d=778514660$, leading to three integral quadruples

(778514660, 3114058640, 1557029320, 778514660),

(778514660, 1907360917, 2847139328, 695102375),

(778514660, 3275183240, 915488420, 1258930960),

with the sum 6228117280 and product $2938712953198523150291392472986880000=$ $2^{11} 5^{4} 7^{4} 11^{4} 17^{4} 131^{4} 227^{4}$.

Example 2. For $n=5$, we have two rational quintuples

$$
(1,1,1,2,5),\left(1,1, \frac{841}{221}, \frac{1690}{493}, \frac{289}{377}\right) \text {. }
$$

Then $d=6409$, leading to two integral quintuples

$(6409,6409,6409,12818,32045),(6409,6409,24389,21970,4913)$,

with the sum 64090 and the product 108131283474484110490. 
Example 3. For $n=6$, we have two rational sextuples

$$
(1,1,1,1,2,6),\left(1,1,1, \frac{1058}{273}, \frac{1323}{299}, \frac{388}{483}\right) .
$$

Then $d=6279$, leading to two integral sextuples

$$
(6279,6279,6279,6279,12558,37674),(6279,6279,6279,24334,27783,4394),
$$

with the sum 75348 and the product 735400878605353561179852 .

\section{Further CONSIDERATion}

The referee asks whether there exists infinitely many $n$-tuples of positive integers with the same sum, the same product, and the same second elementary symmetric function $\sum_{i<j} x_{i} x_{j}$. It's an interesting problem. However, we think it needs a new method even for $n=3$.

\section{ACKNOWLEDGMENT}

The authors want to thank the referee for his or her valuable comments and suggestions.

\section{REFERENCES}

1. L. L. Foster and G. Robins, Solution to Problem E2872, Amer. Math. Monthly, 89(1982) 499-500. MR 1539997

2. R. K. Guy, Unsolved Problems in Number Theory, 3rd edition, Springer Science, 2004. MR2076335(2005h:11003)

3. J. G. Mauldon, Problem E2872, Amer. Math. Monthly, 88(1981) 148. MR1539609

4. A. Schinzel, Triples of positive integers with the same sum and the same product, Serdica Math. J., 22(1996) 587-588. MR1483607 (98g:11033)

5. J. H. Silverman and J. Tate, Rational points on elliptic curves, Springer, 1992. MR.1171452 (93g:11003)

6. T. Skolem, Diophantische Gleichungen, Chelsea, 1950.

Department of Mathematics, Zhejiang University, Hangzhou 310027, People's RepubLIC OF CHINA

E-mail address: zhangyongzju@163.com

Department of Mathematics, Zhejiang University, Hangzhou 310027, People's RepubLIC OF CHINA

E-mail address: txcai@zju.edu.cn 